\title{
Le « monstrueux corporel » : Phèdre de Jean Racine mis en scène par Patrice Chéreau
}

\author{
Marine Deregnoncourt \\ Université Catholique de Louvain, Belgique
}

\section{Introduction}

Du mercredi 15 janvier au dimanche 20 avril 2003 au théâtre parisien de l'Odéon (Declercq 115), ${ }^{1}$ Patrice Chéreau offre, par le biais de sa mise en scène de Phèdre de Jean Racine, une figuration inédite de la femme et de son désir. Phèdre $\mathrm{y}$ apparaît non pas comme un objet désiré mais bel et bien comme un sujet désirant. Cette héroïne mythique incarne véritablement le désir féminin. Comment cela se manifeste-t-il ? De quelle manière représenter la passion impossible et tragique? Autrement dit, comment exprimer ce qui ne peut l'être ? Quel est, dans ce cadre, le rôle du langage ? Comment Patrice Chéreau établit-t-il un rapport entre le corps et le texte ? Que faut-il précisément entendre par «monstrueux corporel»? Où les comédiens doivent-ils se situer et se positionner durant les répétitions ? Dans quel contexte général la démarche artistique de Patrice Chéreau s'inscrit-elle ? Autant de questions que nous nous posons, qui vont nous guider tout au long de cet article et auxquelles nous allons, in fine, tenter de répondre avant de conclure.

\section{Un défi : mettre en scène Phèdre de Jean Racine}

Patrice Chéreau s'est toujours juré de ne jamais mettre en scène un dramaturge français en alexandrins. D'une part, il abhorre la musique de ce type de vers et, d'autre part, il n'apprécie guère le côté figé du théâtre classique français auquel il préfère la sauvagerie shakespearienne. Pourtant, en 1995, à Ivry-sur-Seine, à la fin des répétitions de la troisième version de la mise en scène de la pièce de Bernard-Marie Koltès Dans la solitude des champs de coton, la créatrice des costumes Moidele Bickel met ce metteur en scène au défi de faire entendre Jean Racine comme il y est parvenu avec Bernard-Marie Koltès. En d'autres termes, il s'agit de faire entendre la parole racinienne sur le désir dans le même cadre spatio-temporel et avec les mêmes outils utilisés pour la mise en scène koltésienne sur lesquels nous reviendrons ultérieurement. Patrice Chéreau décide donc d'aborder, pour la première fois, le théâtre racinien. Il choisit Phèdre car cette tragédie s'intéresse autant à la répression du désir qu'à la culpabilité du plaisir. C'est pourquoi, sous le regard de ce metteur en scène, cette tragédie fait à bien des égards écho à Dans la solitude des champs de coton. « La traversée de l'œuvre de Koltès l'avait peut-être préparé à déchiffrer, sous la clarté des chaînes de la syntaxe, la hantise d'une autre face du langage, fuyante, enfouie, indicible, condamnée pourtant à s'ouvrir avec insistance une voie vers le jour $\gg$ (Loayza n.p.).

Fort de ses expériences de metteur en scène du théâtre koltésien et

\footnotetext{
${ }^{1}$ Cette mise en scène a précisément lieu dans les Ateliers Berthier, ancien entrepôt des décors des spectacles de l'Opéra de Paris, qui est alors la salle provisoire du Théâtre de l'Odéon, duquel le site principal est en réfection.
} 
curieux de relever ce défi, Patrice Chéreau relit Phèdre de Jean Racine, la version grecque d'Euripide et latine de Sénèque en se posant les questions suivantes : " Qu'est-ce que j'entends ? Qu'est-ce que je comprends ? Qu'est-ce que je ne comprends pas ?» (Salino n.p.). Ces questions vont lui permettre de mettre au jour une héroïne profondément humaine, charnelle et ravagée par les contradictions du désir, "idée-force» de cette adaptation scénique. Patrice Chéreau s'affranchit ainsi de la «doctrine classique » pour privilégier davantage l'esthétique antique et ce, de deux manières :

- d'un point de vue esthétique et stylistique, les descriptions des récits antiques sont assez crues et explicites ;

- d'un point de vue dramaturgique, Hippolyte apparaît, in fine, sur scène agonisant (Euripide) et le corps mutilé (Sénèque) (Declercq 125).

Patrice Chéreau travaille intensément le texte auquel il est soumis, tout en ne l'étant pas. Pour ce metteur en scène, soumission ne signifie pas respect. Il est soumis à l'écrit et au pouvoir des mots, à leur construction, à leur musique secrète, à leur ouverture sur le monde et sur autre chose. Il s'agit pour lui de provoquer son imaginaire, d'inventer voire de réinventer les mots en analysant le texte. Dès lors, la création théâtrale s'apparente à une spirale qui part du noyau dur du texte exploré dans ses moindres détails avant d'aller vers des sphères davantage complexes liées à l'inconscient. Grâce à des associations d'idées et à des réminiscences qui constituent un arrière-plan nourricier, l'œuvre n'est alors plus isolée de la vie mais rayonne autour d'une énergie vitale. Il s'agit de se soumettre au texte afin de le réinventer et de le redécouvrir, autrement dit «rendre manifeste l'imaginaire latent du texte par l'exploration de son palimpseste mémoriel » (132).

Selon Patrice Chéreau, déclamer le texte n'est pas encore entrer dans le jeu d'acteur qui est avant tout mensonger. La mise en tension entre des pôles oxymoriques, des directions dichotomiques, tout comme la confrontation entre des concepts paradoxaux ou le rapprochement entre des genres a priori étrangers sont les moteurs de cette dynamique.

Le texte de Phèdre que le metteur en scène choisit pour cette mise en scène est tiré de l'édition de 1677 car celui-ci lui permet, tout d'abord, d'enjamber la rime et de ne pas s'arrêter à l'hémistiche. Dans cette édition, la ponctuation est minimale et se limite au point, à la virgule et au point d'exclamation. Patrice Chéreau s'oppose autant à l'arrêt à l'hémistiche (au bout de la sixième syllabe) qu'à l'arrêt à chaque vers. Ensuite, il considère que cette édition textuelle est assez claire et précise quant à la ponctuation. La musicalité de l'alexandrin est ainsi cassée pour privilégier le sens. Il s'agit de suivre les articulations de la syntaxe, conduire la phrase et la maintenir jusqu'au bout de l'intention pour pouvoir ultérieurement libérer le sens dans la pleine résonance, l'écho ou l'écoute du silence. Les mots sont retenus et distillés par des hommes et des femmes qui tombent, dérapent et glissent sur l'alexandrin suivant pour faire vibrer ce type de vers. "J'ai demandé aux comédiens qu'ils tapent sur les mots comme sur les touches d'un instrument pour que jamais deux rimes ne soient dites sur le même ton » (La Bardonnie et Lefort n.p.). Les alexandrins se chevauchent à trois reprises et raisonnent en polyphonie quand quatre personnages sont présents sur scène pour exprimer la même chose, à savoir « partir » ou « fuir ».

Patrice Chéreau donne à voir aux spectateurs le gouffre, l'énigme illimitée et inépuisable du désir féminin irrationnel et amoral. Il octroie ainsi un 
caractère résolument moderne à cette tragédie classique racinienne de désir et de mort. Cette mise en scène tend à puiser dans les racines profondes de cette héroïne mythique pour retrouver le caractère violent du désir, de l'interdit, du dégoût physique et intellectuel de soi. Phèdre entretient un profond rapport de haine envers elle-même. Le plaisir textuel résonne ainsi avec le monde sensible et brûlant qui tend vers la mort. La prise de liberté assumée du metteur en scène par rapport à l'œuvre initiale permet d'en cerner davantage la portée intime et éthique et de faire ici entendre la voix de Phèdre à un public qui ne la connaît pas. Pour ce faire, il n'hésite pas à couper dans la version racinienne afin de décoder le sous-texte, le non-dit, le contenu sociologique et historique.

Cette mise en scène se réfère au désir, à l'interdit et au crime. Ces trois concepts sont cristallisés dans la parole sans qu'il y ait une quelconque action entreprise. Il s'agit précisément d'interroger cette parole puissante car l'inavouable est sous-jacent dans cette tragédie. Comment exprimer par des mots ce qui ne peut être l'être ? C'est la raison pour laquelle Patrice Chéreau privilégie l'aveu - Phèdre étant la tragédie des aveux - et fait figurer, dans ce cadre, le jeune fils de Phèdre à la scène 5 de l'acte II pour le rendre pleinement spectateur de l'obscénité de sa mère laquelle, poitrine dénudée, tourne l'épée d'Hippolyte « vers son corps désirant et infiniment ouvert. Le langage d'agôn, devient agonie et l'amour pur est contaminé par l'amour maudit» (Biet n.p.).

Dans ce spectacle, l'épée est un objet fréquemment utilisé. Dès le début, cette arme apparaît et disparaît. Les protagonistes qui tour à tour la touchent paraissent s'y brûler. L'épée ne sert dès lors pas uniquement à faire accuser Hippolyte. Elle métaphorise un désir refoulé qui ne demande qu'à pleinement s'exprimer.

De toute évidence, Patrice Chéreau abhorre le monologue. C'est la raison pour laquelle un personnage interagit toujours avec un autre partenaire, qu'il soit son ami, son ennemi, un témoin ou un personnage muet. « Sur la scène, un réseau de regards polarise l'action, chaque personnage est témoin de l'autre, spectacle pour l'autre»(La Bardonnie et Lefort n.p). Les protagonistes s'observent, s'affrontent et se fuient. Chaque phrase s'apparente à un acte et le corps est, quant à lui, un lieu de conflit. « Son intuition lui fait percevoir les fils invisibles qui lient Phèdre et Hippolyte, deux pôles (l'un maudit, l'autre pur) d'une même destinée et lutteurs solitaires qui se sentent la proie d'un charme fatal voulu par les dieux » (La Bardonnie et Lefort n.p.). Hippolyte aime Aricie, sœur des Pallantides, fratrie ennemie de son père Thésée. Le jeune homme a honte de son désir et considère ce sentiment comme un manquement à sa ligne de conduite, à savoir l'orgueil d'être pur. Fille de Minos et de Pasiphaé, Phèdre se croit, vu son ascendance, vouée à la fatalité divine d'une malédiction familiale. En effet, elle est convaincue d'être victime de Vénus et qu'il lui faut « demeurer » pour ensuite mieux fuir son beau-fils Hippolyte en mourant. Or, les prisonniers sont libres tout en ne l'étant pas car ils sont captifs de leur imaginaire. La passion amoureuse ne s'apparente pas à une fatalité mais à une absence de volonté. Ce sentiment pourrait sauver les deux personnages mais, à contrario, les étouffent dans une haine du désir qui les conduira inéluctablement à la mort. Comme Jean Racine l'a antérieurement remarqué, Phèdre et Hippolyte sont les deux versants d'un destin semblable. Ils se contaminent l'un l'autre, cèdent à la tentation et osent verbalement exprimer non seulement leur désir mais aussi leur faute. L'un et l'autre, en taisant ultérieurement à Thésée leur face-à-face, deviennent malgré eux complices d'un même secret funeste 
qui amènera le père (Thésée) à tuer le fils (Hippolyte). En effet, la lame de l'épée aura, in fine, raison d'Hippolyte. Le cadavre ensanglanté du jeune homme est exposé aux yeux de tous - spectateurs comme comédiens - sur un plateau de morgue qui provient d'une cage d'ascenseur, tel un crématoire. Patrice Chéreau s'est ici inspiré des Métamorphoses d'Ovide. En tant que lieu de partage et d'articulation entre dire et montrer, entre donner à imaginer et faire voir, entre terreur dramatique et horreur scénique, «Le Récit de Théramène » interroge l'esthétique de la représentation. C'est un récit à la signification équivoque tant en ce qui concerne la réalisation scénographique écartelée entre les arts visuels et oratoires qu'en ce qui concerne l'écriture dramatique, dont le défi est d'émouvoir sans montrer. "Le Récit de Théramène » est le point d'orgue spectaculaire de cette mise en scène et atteste de la dichotomie entre l'esthétique racinienne et chéraldienne. Patrice Chéreau paraît privilégier un signe théâtral « réaliste » duquel la visualité agressive contamine et concurrence le discours de Théramène, quitte à "rabattre le signe sur la chose » (Cavaillé 134). Si le metteur en scène se distancie de Jean Racine, c'est tout d'abord par souci de plaire au public. Afin de toucher les spectateurs, il faut pouvoir blesser l'œil et provoquer l'imaginaire. Imprégnés de culture audiovisuelle et médiatique, les spectateurs sont actuellement davantage adeptes des images que des mots. L'image, cinématographique notamment, participe à la construction de nos représentations mentales. Tout autant que la terreur, l'horreur participe donc grandement à la réception de l'opsis. Ensuite, Patrice Chéreau introduit dans sa mise en scène un dispositif technique moderne (méchané grâce auquel descend le corps et eccyclème qui permet d'exposer ledit corps au regard des spectateurs) pour amener le corps ensanglanté d'Hippolyte. Ce choix octroie une résonance sociopolitique à l'œuvre, située ainsi dans un contexte industriel. Enfin, d'une part, dans l'esthétique chéraldienne, le corps joue un rôle prédominant. Le corps est présence physique désirante, désirée et oppressante. Impudique et intime, le corps saigne et exsude. D'autre part, la confrontation violente est un mode d'interaction récurrent dans la dramaturgie et scénographie chéraldiennes.

\section{Une intrigue en clair/obscur, entre savoir et aveuglement}

Pris dans le huis-clos d'un hangar théâtralisé des anciens entrepôts des décors de l'Opéra-Comique (sur le boulevard parisien des Ateliers Berthier) (Declercq 115), les spectateurs assistent à la chute des « autres », autrement dit de ceux qui expriment la violente irritation de la passion. Le public représente le chœur antique veillant sur le destin funeste des personnages tragiques qui, bientôt s'avanceront au milieu des deux rangées de gradins, « dans l'espace de l'enchaînement fatal des passions et de la mort » (Champs et Lassaign n.p.). L'espace choisi par Patrice Chéreau se voit marqué par sa fonction originelle. Le sol est bétonné et la surface est inégale. Les gradins sont, quant à eux, disposés bi-frontalement, à l'instar du dispositif d'ores et déjà utilisé pour $L a$ Solitude koltésienne.

Les spectateurs à peine installés sont directement plongés in medias res, autrement dit au cœur de l'action par l'arrivée inopinée d'Hippolyte. La quiétude du spectateur doit nécessairement être violée et offensée pour pouvoir entrer dans l'univers fictionnel. Hippolyte annonce à son confident Théramène son désir de fuir et de retrouver son père Thésée lequel est un modèle à ses yeux. Hippolyte, connu pour sa misogynie, brûle pourtant d'une passion dévorante et coupable pour Aricie. Cet amour est en effet coupable car, rappelons-le, cette 
jeune femme est l'ultime descendante des Pallantides, la fratrie ennemie de la famille royale athénienne.

À l'instar d'Hippolyte, Phèdre, dès son entrée en scène, s'impose de réprimer le désir qui provient de sa passion. Tel est le destin fatal auquel elle ne peut échapper qu'en mourant. C'est «le récit fascinant d'un chemin entre la passion amoureuse qui a besoin de hurler et le violent silence de ce désir inavouable » (Loayza n.p.). Phèdre aime Hippolyte. Son âme lutte contre son corps. Cette héroïne est inéluctablement vouée à la nuit. Toutefois, elle souhaite, une dernière fois, voir son beau-fils et le soleil (au sens propre comme au sens figuré). Mais la honte de ses sentiments incestueux provoque la haine envers elle-même et la douleur du secret au moment où les Enfers semblent répandre la rumeur de la mort de Thésée.

Suivant les conseils de sa nourrice Oenone, Phèdre avoue son amour à Hippolyte mais ce «J'aime » (crié) scelle sa destinée avec le retour de son époux, incarnation de la Loi. Phèdre sent déjà les murs l'accuser alors elle laisse à Oenone le soin de calomnier Hippolyte de ce coupable amour. " On l'accuse de ce qu'il n'a jamais fait, parce qu'il s'était juré qu'il ne le ferait jamais : aimer physiquement. Avoir du désir. Il y a une volonté de suicide et de malheur phénoménale chez lui »(Salino n.p.). Après avoir dévoilé le secret de Phèdre, Oenone devient le double noir et chtonien de sa maîtresse -et ne verra d'issue que dans le suicide. Quant à l'héroïne, après avoir rétabli la vérité auprès de son mari Thésée, elle expire ravagée par la honte et par son crime. En censurant avec l'accord des comédiens les derniers vers raciniens, Patrice Chéreau réduit tout espoir à néant. Porté par quelques notes de la Tentation de Saint Antoine de Peter Gabriel, Thésée revient des Enfers revêtu d'un manteau pourpre lequel pourrait symboliser la tentation christique. Trompé par la calomnie d'Oenone, il se croit trahi par Hippolyte et n'hésitera pas à faire appel à Neptune (dieu de la Mer) pour l'occire. Dans le contexte tragique, les pères sont en effet plus forts que les fils et peuvent dès lors les tuer. De fait, la confrontation entre le père et le fils (Acte IV, Scène 2) est d'une véhémence verbale accrue et d'une gestique extrêmement violente. Thésée écrase sous son pied le visage d'Hippolyte et le menace avec un glaive, tel un geste sacrificiel. Néanmoins, après avoir attentivement écouté le récit de Théramène relatant la mort de son fils, Thésée se sent coupable de ses erreurs. Pour autant, contrairement à la version racinienne, il n'adoptera pas Aricie. Une chanson de Prince résonne alors soudainement à la fin de la pièce, comme pour accentuer et renforcer le caractère tragique du personnage de Thésée. En effet, le roi exprime ses remords et sa douleur de père mais restera seul dans son labyrinthe. Dès lors, nous pouvons constater que l'accompagnement musical souligne et sert le propos véhiculé par Patrice Chéreau dans sa mise en scène. En effet, la musique participe à la chorégraphie chaotique qui provient de la dérive intérieure de chaque personnage. Ce sont les passages lyriques - surnommés « tableaux » par Roland Barthes - qui sont appuyés par la musique, autrement dit ces moments où les personnages sont sur un fil qui pourrait à tout instant se briser. Le fantôme de l'Opéra règne sur ce spectacle. Patrice Chéreau précise qu'avoir mis en scène des opéras lui a fait remarquer que la langue racinienne s'apparente à une succession d'arias et de silences. Dès lors, une réplique n'est pas spontanée. Elle jaillit à la suite d'une réaction ou d'une idée, sans pour autant l'expliciter intelligiblement.

\section{Le choix des comédiens}


Patrice Chéreau opte pour une division des personnages qui s'apparente à une relique du chœur antique. Trois corps masculins - incarnation des trois âges de la vie - rentrent en conflit avec cinq monstres femelles.

«Visage si ovale, yeux ronds, épaules tendres de jeune mère capable de plier son jeune fils à ses fins, pire que folle parce qu'extra-lucide et au fond très calme, y compris dans le suicide. Dominique Blanc ... est ... la Phèdre absolue » (La Bardonnie n.p.). D'une part, Dominique Blanc définit Phèdre comme une héroïne "combustible », prête à se laisser détruire et consumer. D'autre part, elle prétend aussi que son personnage est « une gigantesque amoureuse, une amoureuse majuscule » en proie à une grande violence, sentiment normalement réservé aux hommes. "C'est un rôle dont on rêve toutes. Phèdre fascine car elle incarne l'extrême désir féminin qui, souvent, reste incompris des hommes" (Champs et Lassaigne n.p.). La comédienne a rencontré des difficultés pour se détacher de son personnage car incarner sa propre mort durant trois mois demande de puiser dans des fondations, des soubassements et des racines (sans jeu de mots) intimement enfouis. Elle a pris autant de plaisir à incarner ce rôle qu'à en être dévastée : « Il y a eu une vraie rencontre entre le personnage, la pièce, le metteur en scène et moi-même » (Pailloux-Riggi 163). Phèdre lui a appris, par sa quête d'amour absolu, à aimer. C'est un personnage qui, selon elle, grandit et fait grandir. Par conséquent, l'interprète a dû puiser dans ses forces et simultanément se laisser déborder pour laisser le corps sans limites exprimer pleinement les contradictions du rapport de son personnage au monde.

En ce qui concerne les tenues scéniques, elles participent à la bonne attitude des comédiens. Elles sont conçues sur mesure et fonctionnent en harmonie avec les personnages. Par conséquent, les vêtements portés font corps avec les comédiens et participent à leur métamorphose. Les acteurs portent des capes d'étoffe ou des robes aux couleurs tranchées qui contrastent avec la blancheur tentatrice de la chair : bleu (Hippolyte et Aricie), noir (Phèdre) et rouge (Thésée).

Phèdre arrache ses vêtements serrants tandis qu'Oenone, incarnée par Christiane Cohendy, apparaît avec des cheveux gris, des chaussures de bonne sœur et vêtue d'étoffes de laine de couleur brune. Le personnage de la nourrice est un personnage tant actif que désolé et désolant, à la fois mère et servante. Panope interprétée par Nathalie Bécue n'apparaît que brièvement sur scène pour annoncer la mort de Thésée. Ismène, quant à elle, incarnée par Agnès Sourdillon, ne parle guère mais voit et comprend néanmoins tout de l'intrigue.

Marina Hands interprète Aricie, jeune femme pure, candide, vêtue de lin gris et d'un manteau bleu. " La captive Aricie » est tout d'abord une sœur pour Hippolyte avant d'être, par sa manière de le regarder et de l'enlacer, son amante. Marina Hands prétend avoir confiance en Jean Racine; auteur qui pourtant ne confère à son personnage que peu de temps de parole. C'est pourquoi Aricie est fréquemment considérée comme un rôle secondaire et de moindre importance. La comédienne se définit comme une « interprète » et un " instrument 》 au service du dramaturge classique duquel elle se sent proche et dont elle tente de comprendre le discours. Dans la mise en scène de Patrice Chéreau, Aricie existe à un point tel que le personnage et son incarnation ne semblent faire qu'un. Quant au public, il suit pas à pas le parcours elliptique de l'héroïne. Sur scène, Marina Hands se métamorphose et éprouve une nécessité vitale de jouer. « J'adore ce moment d'appropriation où le texte passe par le corps et où l'on a l'impression que l'interprète a parlé en alexandrins toute sa vie » (Diatkine n.p.). 
Le rôle d'Hippolyte est, quant à lui, attribué à Éric Ruf, sociétaire et depuis 2014 administrateur général de la Comédie-Française. Cet acteur louange l'intuition et le « savoir aérien » de Patrice Chéreau qui intensifie la prégnance des désirs pour en définir la présence froide et sanglante dans un grand atelier contemporain (Biet 3). Dominique Blanc approuve ce choix de comédien. " Pour une fois, Phèdre n'a pas en face d'elle un androgyne de seize ans, mais un homme, et on peut comprendre le désir qu'elle éprouve. Il y a dans cette distribution un parti pris de sensualité extrêmement fort, dans ce spectacle une façon guerrière de décrasser le chef d'œuvre » (Godard 257).

Aux côtés de Dominique Blanc, d'Éric Ruf, de Marina Hands, de Christiane Cohendy, de Nathalie Bécue et d'Agnès Sourdillon, Michel Duchaussoy incarne « la noblesse émouvante de Théramène » (260). En ce qui concerne la figure du père, « dont l'absence pèse comme un remords, dont la présence frappe comme un châtiment divin » et qui précipite l'inévitable funeste fin, il est interprété par Pascal Greggory (260). Ce comédien interprétait auparavant le rôle du client dans l'ultime mise en scène de Patrice Chéreau de la pièce koltésienne Dans la solitude des champs de coton. Dans ce cadre, Pascal Greggory donnait la réplique à Patrice Chéreau, lequel incarnait le dealer. Du théâtre koltésien à la tragédie racinienne, il n'y a vraisemblablement qu'un pas. Par le biais de ce rôle, Pascal Greggory scelle dans le sang d'Hippolyte, avec lequel il se scarifie le visage, l'union de Jean Racine à William Shakespeare.

À travers le corps ensanglanté, c'est un sentiment violent et refoulé, contenu intrinsèquement dans le corps, qui se révèle par les pores de la peau. C'est l'ultime réaction physiologique d'un désir qui ne peut plus supporter son étroitesse et son écart par rapport au monde. Le sang sacralise la tension entre l'extériorité et l'intériorité des personnages (intimité, honte ou pudeur), à tel point que, sous le regard d'autrui, le premier terme devient une excroissance du second. Le sang montré ainsi au public est une pratique fréquente chez les Grecs que Patrice Chéreau reprend dans cette mise en scène. Il souhaite en effet retrouver le théâtre antique dans la tragédie classique française de Jean Racine, auteur «qui, bien qu'il n'y consente, s'est autant inspiré de Sénèque $\neg$ deux scènes mot pour mot $\neg$ que d'Euripide. C'est-à-dire revenir à la légende, à des sources qui sourdent depuis l'origine du monde. Voilà mon dessein. Raconter cette histoire aux gens. Trouver la possibilité de faire parvenir ce texte étrange et ce qu'il véhicule aujourd'hui » (La Bardonnie et Lefort n.p.).

Quel que soit le comédien, il est d'une part écorché, malmené ou agressé par ses partenaires, rampant et se traînant à terre avec la peau à vif. D'autre part, il est sans cesse plié en deux. "C'est physique. La passion fait mal au ventre. On se tord de douleur à désirer » (La Bardonnie et Lefort n.p.). Le metteur en scène préfère que les comédiens soient accroupis plutôt qu'en position verticale et affirme que la composition d'un rôle débute avec la façon dont les acteurs se tiennent sur le plateau.

\section{Un décor antique et épuré}

Le plateau est vide, les costumes sont sobres et les lumières sont crues. Une muraille de pierre élaborée par Richard Peduzzi s'élève vers les dieux, "vertigineuse falaise sculptée comme l'entrée d'un palais ou celle d'un tombeau » (Champs et Lassaigne n.p.). C'est autrement dit un no man's land et une architecture antique inspirée des temples et tombeaux de Pétra du Bernin. En outre, une passerelle venant d'outre-tombe, contemporaine et incertaine, sur 
laquelle Phèdre vacille, relie cette entrée antique du palais royal athénien à un plateau court, étroit, étiré et rectangulaire qui fait face aux spectateurs installés bi-frontalement. C'est un dispositif auquel Patrice Chéreau a déjà, rappelons-le, eu recours dans ses mises en scène des pièces koltésiennes, Combat de nègre et de chiens et Dans la solitude des champs de coton. D'une part, le metteur en scène prétend que ce dispositif permet d'allonger la distance de jeu pour arriver à une vision panoramique, à l'instar de la distance entre Phèdre et Théramène quand ce dernier décrit la mort d'Hippolyte. D'autre part, ce dispositif permet aussi un face à face grâce auquel chacun se tourne autour, tel un mouvement rotatif. Les spectateurs peuvent ainsi plus aisément voir les yeux des comédiens. Autrement dit, ce type de dispositif déstabilise le theatron. Le spectateur est autant regardant que regardé et les acteurs sont vus de tous côtés. La frontière est dès lors ténue entre espace spectatorial et espace scénique. Patrice Chéreau apprécie que les spectateurs perçoivent les larmes, la transpiration et l'intimité des comédiens. Le public domine la scène et forme une assemblée. Par conséquent, cette mise en scène est proche des gens. L'essentiel pour Patrice Chéreau, c'est un texte et des corps dans un espace en lien direct avec le public. En tant que successeur de Berthold Brecht, Patrice Chéreau considère que le public est le destinataire phare de ses réalisations artistiques.

\section{Les lumières, dispositif théâtral en adéquation avec le propos}

Le plateau est plongé dans une semi-obscurité bleutée avant que les acteurs ne soient saisis par de fortes et brusques douches de lumière. Tous les personnages sont véritablement poursuivis par la lumière au point d'en devenir captifs. Les trois poursuites issues du dispositif utilisé pour Dans la solitude des champs de coton de Bernard-Marie Koltès découpent l'espace réservé aux acteurs. Par conséquent, les machinistes sont très actifs. Les acteurs deviennent les proies d'un destin accablant et étouffant. Ils devront tenter le pas de côté qui les éloignerait d'un rôle auquel ils ne peuvent se soustraire avant d'être à nouveau saisis par la froide lumière. L'éclairage s'harmonise à la mise en scène, à la thématique traitée par le spectacle et produit les « effets » voulus par l'action. Dès lors, la lumière fait partie intégrante du spectacle et conditionne non seulement le travail de mise en scène mais aussi le jeu des comédiens.

Après l'invocation de Thésée au dieu Neptune, a lieu hors scène la rencontre entre Hippolyte et le monstre marin. Un monte-charge amène sur scène, rappelons-le, le cadavre ensanglanté du jeune homme. La poursuite étant arrivée à sa fin, la lumière devient alors plus chaude, teintée de larmes et de compassion. Hippolyte apparaît dénudé et pris dans une pietà coupable qui entremêle bave et sang pur du martyr. Cette tragédie de la lumière est alors centrée sur Hippolyte, jeune homme objet de toutes les passions, déchiré entre ses contradictions et sa culpabilité.

\section{«Organiser le secret » (Chéreau De Sartrouville 56) : le rapport de Patrice Chéreau à ses comédiens}

Patrice Chéreau entend rapprocher un personnage tragique d'une problématique existentielle à laquelle chacun doit faire face. Les comédiens cassent ainsi les clichés généralement associés à leurs personnages et sont entièrement tournés vers un affect complexe. Pour ce faire, le metteur en scène observe les acteurs avec qui il travaille. Il est attentif aux impulsions qui 
jaillissent de leur for intérieur. Par ses mots, il a envie de faire frémir leur âme pour qu'ils dessinent corporellement sur scène « les profondeurs furtives des êtres » (Benhamou 16). Il désire que toute l'émotion contenue dans le spectacle passe par eux. Tel un chef d'orchestre dont il adopte la gestuelle et l'attitude, Patrice Chéreau accompagne physiquement les comédiens dans leur jeu en étant présent sur le plateau. C'est pourquoi les répétitions sont importantes à ses yeux car elles sont un perpétuel échange à travers lequel les acteurs et le metteur en scène se nourrissent pour faire naître un « sous-texte » qui doit colorer le texte et le projeter en acte. Ce «sous-texte » peut, soit être élaboré avec Patrice Chéreau, soit demeurer le secret des comédiens. Cette première mise en bouche et en corps est une étape de mise à l'épreuve en coopération avec l'acteur dans le seul but de faire entendre l'auteur et de suivre son cheminement souterrain.

Patrice Chéreau définit son travail de mise en scène comme un premier regard posé qui est à ses yeux essentiel. « Diriger les acteurs ne veut pas dire les utiliser, mais provoquer en eux la naissance de quelque chose, les modifier, faire que l'émotion du spectacle passe par eux, par la rareté de leur nature, à leur insu ou non. ... si le travail avec les comédiens ne va pas en profondeur, si le metteur en scène ne provoque pas leur imagination instinctive et si les comédiens ne sont pas de très grands exécutants, il ne se passera rien sur le plateau !» (Benhamou 49). ${ }^{2}$

Si Patrice Chéreau est aussi exigeant, c'est parce qu'il attend beaucoup de la part de ses acteurs. Pour lui, l'acteur rêvé est celui qui révèle ses parts d'ombre. La « présence » du comédien est liée à un questionnement éthique. L'acteur doit être transcendé par son rôle, lequel doit agir sur lui. C'est ainsi que le comédien est propulsé dans des zones de jeu où il est dépouillé de son savoir-faire. Selon Patrice Chéreau, c'est fondamental qu'il se passe quelque chose lors des représentations.

Comme « organiser le secret» revient à approfondir inlassablement la distance paradoxale entre le langage et l'affect, les comédiens doivent faire appel à des ressources profondément enfouies. Cette introspection renvoie à la vérité de l'« entre » des mots et de l'instinct. Selon Patrice Chéreau, cette conception répond à celle du sujet en tant qu'il est divisé entre ses pulsions et le langage, incapable d'être authentique car il échappe sans cesse à lui-même. " Organiser le secret » revient à placer sur cette faille - par laquelle passera subrepticement une forme de vérité - le personnage et l'acteur dont le jeu provient du décentrement, du tréfonds et du déchirement. Les personnages qui composent le monde de Patrice Chéreau sont embrigadés dans leur passion amoureuse, érotique ou leur narcissisme et le réel se répercute inévitablement sur eux. Tout être humain désirant ou entièrement tourné vers le plaisir est inéluctablement sacrifié et broyé.

Afin de rendre ces émotions vivaces sur scène, les comédiens se réunissent en huis clos, dans l'intimité et surtout pas en public car la parole délivrée serait inévitablement différente. En effet, pour le metteur en scène, la captation filmique dénonce les artifices du théâtre, à savoir la voix, les bruits et le texte. Dès lors, il préfère que les caméras participent au making-of, autrement dit à la création en train de se faire. Les acteurs s'écoutent mutuellement, lisent leur rôle «à la table » et commencent à jouer. Patrice Chéreau est présent pour élucider le sens en cas de besoin. S'il lui arrive d'indiquer le rôle voire de

2 «La mousse, l'écume. » Entretien avec Émile Copfermann. Travail théâtral 11 (printemps 1973): 14 . 
l'interpréter pour le montrer et ainsi aider les acteurs, il les laisse néanmoins s'approprier, par leur jeu, le texte et ainsi donner chair à leur personnage. Les comédiens expriment alors leur rôle et se révèlent à eux-mêmes. Avec une ou deux idées directrices, le metteur en scène les lance sur une première voie. Ensuite, les comédiens commencent à réagir à l'unisson. Une entente se crée alors et Patrice Chéreau "nourrit » les acteurs de gloses. Au cours de ces sessions de lecture, tous recherchent l'enjeu d'une scène, le parcours accompli par un personnage et la façon dont ce dernier en sort changé et modifié. Le metteur en scène fait fréquemment travailler des scènes isolément avec un ou deux comédiens pour diriger plus intimement le fil conducteur d'un rôle et aller encore plus loin. Les transformations et les conflits se décident à ce moment-là. Les acteurs devront mettre cela en pratique lors des répétitions sur le plateau.

\section{l'allégorie}

« Raconter des histoires », le projet dramaturgique d'un théâtre de

Patrice Chéreau souhaite « raconter des histoires », autrement dit partager l'expérience du réel et « rendre crédible » ce qu'il a envie de voir ou les gens desquels il désire percer le secret. En effet, il veut faire sentir, dans les êtres, ce qu'ils cachent et surtout ce qu'ils se dissimulent à eux-mêmes en creusant « des abîmes entre les mots et les corps » (Benhamou 10), en faisant voir le sens caché des images et en piégeant, au sein des récits, cette part de réel qui se définit comme une faille, à savoir le rapport au monde, le sexe et le désir. Â ses yeux, le désir est sexuel et intrinsèquement lié à la mort.

Les histoires que Patrice Chéreau entend raconter peuvent non seulement contenir le monde mais aussi les difficultés auxquelles les êtres humains sont confrontés ainsi que leur manière d'être au monde pour démontrer la façon dont les gens gèrent ou pas leur vie et peinent à vivre ou à mourir. Tout comme Berthold Brecht, la narration est, pour Patrice Chéreau, vraisemblablement la seule façon de partager le réel et de ne pas demeurer seul face à l'énigme du monde.

En tant que matrice de son travail cinématographique, de mise en scène et de ses récits, Patrice Chéreau invoque sans cesse le manque, la perte, la disparition, l'absence et l'impossibilité (d'écrire). Toutefois, il insiste sur l'envers positif de ces termes, à savoir le plaisir de travailler, le désir réactivé et l'énergie toujours renouvelée. La frustration est au cœur de sa réflexion. C'est la raison pour laquelle ses réalisations (dé)montrent comment se dérobent au désir la pleine jouissance de l'amour, du pouvoir, de la fusion avec autrui, de l'innocence de l'érotisme et de la connaissance de soi. En d'autres termes, il entend explorer l'être de l'homme comme une surface qui sépare la région du semblable de celle de l'altérité. Sous les instincts de conservation et de plaisir, il existe Éros (l'instinct de vie) et Thanatos (l'instinct de mort) qui planent sur son œuvre marquée par la peur de la fin de la vie réelle, sociale ou amoureuse.

La réception critique de ce metteur en scène et cinéaste se caractérise par une vision tragique du rapport de l'être humain au monde. Or, son œuvre est éloignée de la catharsis. Que ce soient ses pièces de théâtre ou ses films, ils encouragent le désir. L'échec des personnages n'est pas présenté comme tel mais « comme la forme inéluctable que prend l'aventure de vivre » (Benhamou 9). Il s'agit d'aimer la surprise livrée par la réalité. Le résultat de cette expérience est fréquemment cruel mais la douleur exacerbée donne l'impulsion aux aspirations des êtres humains, à leur entêtement, à leur libre-arbitre et à leur 
envie de vivre. Il est cependant impossible de trouver ce qui comblera leur manque. Tout ce qu'ils découvrent, c'est l'insolubilité de leur propre désir. Il leur est seulement possible d'accéder à eux-mêmes et à leurs contradictions en se confrontant au réel et au monde.

L'attrait de Patrice Chéreau pour la subjectivité, les affects et les pulsions est indissociable de sa passion pour la réalité. Il entend vivre au présent sans se soucier du passé, du futur, de la nostalgie ou des promesses. Son théâtre met en exergue l'impossibilité amoureuse, l'échec, le deuil et la mort (concept à la fois proche et éloigné de l'individu) ; autant d'antidotes contre la mélancolie. Ce metteur en scène s'intéresse également aux faiblesses, aux blessures enfouies et à l'inassouvissement. Ce souci du réel renvoie à la perspective réaliste, telle qu'elle est définie par Berthold Brecht, à savoir « qui dévoile la causalité complexe des rapports sociaux ; qui dénonce les idées dominantes comme les idées de la classe dominante ; qui écrit du point de vue de la classe qui tient prêtes les solutions les plus larges aux difficultés les plus pressantes dans lesquelles se débat la société humaine ; qui souligne le moment de l'évolution en toute chose ; qui est concret tout en facilitant le travail de l'abstraction » (De Sartrouville 41). Patrice Chéreau définit son théâtre comme un art de l'allégorie, autrement dit un art de la monstration capable de figurer le sens et de faire devenir visible l'abstraction du drame. Il s'agit d'inventer des images dialectiques, vraisemblablement en référence à l'Origine du drame baroque allemand de Walter Benjamin. Ce philosophe définit l'allégorie en opposition à l'unité « du sensible et de l'intelligible » du symbole romantique. Pour Walter Benjamin, l'allégorie se refuse à opérer une fusion ou une synthèse. Au contraire, c'est un concept qui n'a de cesse de manifester une tension entre la forme et le sens. Le théâtre de Patrice Chéreau en est caractéristique car, d'une part, il tend vers l'authenticité de la vie sur scène et d'autre part « vers la netteté d'un contenu critique » (Benhamou 35). Contrairement au symbole, l'allégorie vise à révéler le particulier dans l'universel. En effet, elle précipite la signification dans un personnage, un objet ou une image. En incarnant l'idée la plus abstraite (violence du fonctionnement du pouvoir, perversion des jeux du désir) dans le concret (matériau corporel et pulsionnel, matière d'un sol ou d'un mur, la lumière ou l'étrangeté d'un lieu), Patrice Chéreau entend « ouvrir des brèches vertigineuses dans la reconnaissance du réel et dans le naturalisme revendiqué du jeu ; à créer dans la surface du visible un appel vers la profondeur » (De Sartrouville 33, 41). Le projet figuratif du théâtre de Patrice Chéreau «consiste à organiser sans relâche, par des déplacements, des contradictions, des paradoxes, des renversements, des retournements, un hiatus au cœur des images, où s'incarne le drame» (Benhamou 35). D'ailleurs, le metteur en scène avoue qu'il entretient une relation viscérale avec les images et leur production. Il existe de fait un hiatus entre l'opacité corporelle et la lisibilité d'une lutte ou l'inéluctabilité du désir, entre la matière d'un lieu et l'idée induite par ce lieu, entre le caractère brutal et sourd d'un geste et la signification de la composition d'un tableau.

Le surgissement soudain du réel sur scène affirme la permanence d'un ailleurs poético-philosophique qui l'habite et lui confère sa force et sa profondeur. Patrice Chéreau désire « faire de la scène le lieu d'une magnificence plastique » en même temps qu'un monde dont la Vérité repose sur une dépense d'énergie, sur l'animalité corporelle et sur la violence matérielle. Cela entraîne inévitablement, pour le public une expérience paradoxale et divisée oscillant entre manque et plénitude. Dominique Blanc affirme d'ailleurs que « le public 
se sent concerné par une histoire tragique, cela le touche et renvoie à l'expérience de chacun » (Pailloux-Riggi 165). Le metteur en scène oppose aux corps immobiles de la salle, l'humanité qui surgit sur scène basée sur des sentiments confus, sur l'asservissement corporel mystérieux, sur l'aveuglement pulsionnel et sur l'instinct. Cette opposition radicale génère « un abîme sensible pour les spectateurs » (Benhamou 48) en leur renvoyant si violemment cet écart entre la conscience et les actes que cela les émeut, comme si le caractère intense « d'un voir et d'un comprendre » (48) ne peut que se produire qu'en faisant fi des corps. Patrice Chéreau contraint ainsi l'être humain à entreprendre une quête et à renouer avec des problèmes qui sont censés être profondément enfouis.

\section{Conclusion}

«L'égarement à aimer ... est aussi monstrueux que l'injustice dans l'esprit» (Pascal). En atteste cette mise en scène axée sur le caractère «monstrueux corporel» du désir passionnel féminin généré par le langage, arme contre soi et contre ses propres affects. En tant que lieu de conflit et de violence, le corps se métamorphose en monstre. Patrice Chéreau transpose ainsi non seulement le désir féminin qui fait vaciller l'ordre patriarcal mais exprime aussi l'inexprimable, autrement dit une passion tragique inavouable au sein d'un vaste atelier contemporain.

\section{Bibliographie}

Benhamou, Anne-Françoise. Patrice Chéreau. Figurer le réel. Paris : Les Solitaires intempestifs, 2015.

Biet, Christian. «Phèdre (mises en scène C. Rist et P. Chéreau). » Encyclopcedia Universalis [en ligne], 14 octobre 2016.

Cavaillé, Fabien. " "Au théâtre, on n'immole pas." Violence et théâtralité dans le Titus Andronicus de Daniel Mesguich (1989-1992). » Daniel Mesguich. Reprise et transmission. Mireille Calle-Grüber, Stella Spriet, Gilles Declercq (dir.). Presses de la Sorbonne Nouvelle 2012. 127-139.

Champs, Frédérique et Lassaigne, Vanessa, Phèdre. Dominique Blanc. Arte, $1^{\mathrm{er}}$ novembre 2016 [Article PDF].

Chéreau, Patrice. Chéreau. De Sartrouville à Nanterre. La Dispute. Peer Gynt. Les Paravents. Le théâtre lyrique. Paris : Éditions du Centre National de la Recherche Scientifique, 1986. 
---. Les visages et les corps. Paris : Réunion des musées nationaux, 2010.

Declercq, Gilles. « Chéreau/Racine : l'improbable rencontre. » Euvres et Critiques vol. XLI 1 (2016), 115-135.

Diatkine, Anne. « Marina Hands, la captive de la scène. » Libération, $1^{\text {er }}$ novembre 2016.

Godard, Colette. Patrice Chéreau : un trajet. Monaco : Éditions du Rocher, 2007.

La Bardonnie, Mathilde. "Ce "Phèdre" sidère. » Libération, 8 novembre 2016.

La Bardonnie, Mathilde et Lefort, Gérard. « Je connais des gens comme elle. » Libération, $1^{\text {er }}$ novembre 2016.

Loayza, Daniel. «Phèdre de Jean Racine avec Dominique Blanc, Pascal Greggory, Eric Ruff et Michel Duchaussoy dans une mise en scène par Patrice, Chéreau. » A la lettre, $1^{\mathrm{er}}$ novembre 2016.

Pailloux-Riggi, Sophie. Phèdre. Paris : Nathan, 2012.

Pascal, Blaise. Discours sur les passions de l'amour (1652-1653). Paris : Fontemoing, 1900.

Salino, Brigitte. « Chéreau : Pourquoi et comment monter Phèdre de Racine [2003]. » Le Monde, 14 octobre 2016. 